\title{
ON THE JACOBIAN CONJECTURE
}

\author{
SHULIM KALIMAN
}

(Communicated by Louis J. Ratliff, Jr.)

\begin{abstract}
We show that the Jacobian conjecture can be reduced to a weaker conjecture in which all fibers of coordinate functions are irreducible.
\end{abstract}

\section{INTRODUCTION}

Let $(x, y)$ be a coordinate system in $\mathbf{C}^{2}$. The Jacobian Conjecture says that a polynomial mapping $(p, q): \mathbf{C}^{2} \rightarrow \mathbf{C}^{2}$ whose Jacobian $J(p, q)=\partial p / \partial x$. $\partial q / \partial y-\partial p / \partial y \cdot \partial q / \partial x$ is equal to 1 is invertible. This conjecture first appeared in $[\mathrm{K}]$, and one can read a nice survey of the results that concentrated around the conjecture in $[\mathrm{BCW}]$. Recall that a polynomial fiber is reducible if it is the union of more than one algebraic curve. Under the assumptions of the Jacobian conjecture, the system $\partial p / \partial x=\partial p / \partial y=0$ has no solution. From this it follows that different components of a reducible fiber of $p$ do not intersect and the polynomial $p$ has no multiple fiber. Nontrivial polynomials of this kind with reducible fibers exist and $x(x y+1)$ is the simplest example. We shall formulate a new problem.

Weak Jacobian Conjecture. Let $(p, q): \mathbf{C}^{2} \rightarrow \mathbf{C}^{2}$ be a polynomial mapping with $J(p, q) \equiv 1$. Suppose that for every $c \in \mathbf{C}$ the fiber $\{(x, y) \mid p(x, y)=c\}$ is irreducible. Then the mapping $(p, q)$ is invertible.

In other words, the additional condition on the polynomial fibers means that for every $c \in \mathbf{C}$ the polynomial $p(x, y)-c$ is prime. Our main result is the following

Theorem. If the Weak Jacobian Conjecture is true then the Jacobian Conjecture is true.

In order to prove this fact for each couple of polynomials $(p, q)$ with $J(p, q)$ $\equiv 1$ we shall find a polynomial automorphism $\alpha=\left(\alpha_{1}, \alpha_{2}\right): \mathbf{C}^{2} \rightarrow \mathbf{C}^{2}$ such that $p_{1}=\alpha_{1}(p, q)$ does not have reducible fibers. We would like to note also that in order to prove the Weak Jacobian Conjecture one may try to show that a polynomial whose fibers are irreducible and different from $\mathbf{C}$ must have a fiber with a singular point. The last fact holds if this polynomial is good at infinity [NR]. Application of the current theorem enables us to simplify the proof of

Received by the editors December 24, 1990 and, in revised form, May 24, 1991.

1991 Mathematics Subject Classification. Primary 14E07, 13B10, 14 E20. 
the results in [S1-S3], since the existence of reducible fibers makes the original proof more complicated. Recall an equivalent formulation of the Jacobian Conjecture: if $J(p, q) \equiv 1$ then the ring $\mathbf{C}[p, q]$ generated by $p$ and $q$ coincides with the polynomial ring $\mathbf{C}[x, y]$.

The following fact is the two-dimensional case of a theorem in [F] (it is a direct corollary of [W] as well).

Theorem A. If $J(p, q) \equiv 1$ and there exists a polynomial $h$ such that $\mathbf{C}[p, q, h]$ $=\mathbf{C}[x, y]$, then $\mathbf{C}[p, q]=\mathbf{C}[x, y]$.

Combination of our main result and Theorem 3 from $[R]$ gives another proof of Theorem A.

\section{Proof of Theorem}

Let $P: M \rightarrow \mathrm{C}^{2}$ be a regular mapping from a smooth connected algebraic surface $M$ to $\mathbf{C}^{2}$. Suppose that the dimension of the inverse image $P^{-1}(w)$ of every point $w \in \mathbf{C}^{2}$ is equal to 0 . Then there exists an affine algebraic curve $\Gamma \subset \mathbf{C}^{2}$ such that for every $w \in \mathbf{C}^{2}-\Gamma$ the inverse image $P^{-1}(w)$ consists exactly of $n$ points (these points are not multiple). We shall say that $\Gamma$ is the branch curve of $P$.

Definition. We shall say that an irreducible curve $G$ is " $\Gamma$ proper," if the embedding $i: G-\Gamma \rightarrow \mathbf{C}^{2}-\Gamma$ generates an epimorphism $i_{\star}: \pi_{1}(G-\Gamma) \rightarrow \pi_{2}\left(\mathbf{C}^{2}-\Gamma\right)$ from the fundamental group $\pi_{1}(G-\Gamma)$ to the fundamental group $\pi_{1}\left(C^{2}-\Gamma\right)$.

Lemma 1. Let $G$ be a smooth irreducible affine algebraic $\Gamma$ proper curve. Then the inverse image $G^{1}=P^{-1}(G)$ is an irreducible curve in $M$.

Proof. Let $w \in \mathbf{C}^{2}-\Gamma$ and let $P^{-1}(w)=\left\{z_{1}, \ldots, z_{n}\right\}$. Since $M$ is connected, there is a path $s_{i j}:[0,1] \rightarrow M-P^{-1}(\Gamma)$ for which $s_{i j}(0)=z_{i}$ and $s_{i j}(1)=z_{j}$. Put $\tau_{i j}=P \circ s_{i j}$. Then $\tau_{i j}$ is a loop and $\tau_{i j}(0)=\tau_{i j}(1)=w$. This loop generates an element $\left[\tau_{i j}\right]$ of the fundamental group $\pi_{1}\left(\mathbf{C}^{2}-\Gamma, w\right)$. Let $\tau_{i j}^{\prime}$ be another loop that generates $\left[\tau_{i j}\right]$. By the homotopy lifting theorem [FR, Chapter 4] $P^{-1}\left(\tau_{i j}^{\prime}\right)$ contains a path that connects the points $z_{i}$ and $z_{j}$. Let $S^{1}$ be the set of singular points of $G^{1}$. Then the curve $G^{1}$ is irreducible if and only if the set $G^{1}-S^{1}$ is connected. Suppose that $w \in A=G-\left(\Gamma \cup P\left(S^{1}\right)\right)$. Since $i_{\star}: \pi_{1}(G-\Gamma, w) \rightarrow \pi_{1}\left(\mathbf{C}^{2}-\Gamma, w\right)$ is an epimorphism, we can choose all the loops $\left\{\tau_{i j}\right\}$ so that $\tau_{i j} \subset G-\Gamma$. Moreover, by perturbing these loops, we may suppose that $\tau_{i j} \subset A$. In order to prove that $G^{1}-S^{1}$ is connected it is enough to present a path that connects the point $z_{1}$ with an arbitrary point $z^{\prime} \in G^{1}-S^{1}$. Let $w^{\prime}=P\left(z^{\prime}\right)$. Since $A$ is connected, there is a path $\tau:[0,1] \rightarrow A$ with $\tau(0)=w$ and $\tau(1)=w^{\prime}$. Then the inverse image $P^{-1}(\tau)$ contains a path that connects $z^{\prime}$ with $z_{j}$ for a certain $j$. But $z_{1}$ and $z_{j}$ are connected by $s_{1 j}$. This implies the desired conclusion.

The following fact is obvious.

Lemma 2. Let $\left\{\gamma_{i}^{t}:[0,1] \rightarrow \mathbf{C}^{2}-\Gamma\right\}$ be a family of loops in $\mathbf{C}^{2}-\Gamma$ that depend 
Lemma 3. Let $f(c, u, v)$ be a polynomial in $\mathbf{C}^{3}$. Let $F_{0}=\left\{(u, v) \in \mathbf{C}^{2}\right\}$ $f(0, u, v)=0\}$. Assume $F_{0}$ contains a smooth irreducible component $E$ so that either $\partial f /\left.\partial u\right|_{E}$ or $\partial f /\left.\partial v\right|_{E}$ does not equal 0 identically. If the curve $E$ is $\Gamma$ proper, then there exists a neighborhood $U$ of the origin in $\mathbf{C}$ such that for every $c \in U$ the curve $F_{c}=\left\{(u, v) \in \mathbf{C}^{2} \mid f(c, u, v)=0\right\}$ contains a component that is $\Gamma$ proper.

Proof. Choose loops $\gamma_{i}^{0}:[0,1] \rightarrow E-\Gamma$ with $w_{0}=\gamma_{i}^{0}(0)$ for every $i$ such that these loops generate the group $\pi_{1}\left(\mathbf{C}^{2}-\Gamma, w_{0}\right)$. Consider $K_{0}=\bigcup_{i} \gamma_{i}^{0}$. Since the manifold $\mathbf{C}^{2}-\Gamma$ is an algebraic variety, its fundamental group is finitely generated (which follows from $[Z]$ as well). Thus we may suppose that $T$ is compact. Without loss of generality, consider only the case when $\partial f / \partial v$ is not identically zero on $E$. Since $E$ is smooth, one can perturb $\gamma_{i}^{0}$ a little so that $\partial f / \partial v$ is different from zero at every point of $T$. Let $V_{0}$ be a sufficiently small neighborhood of $T$ in $\mathbf{C}^{2}-\Gamma$. Choose a sufficiently small neighborhood $U$ of the origin in $\mathbf{C}$, and put $V^{1}=\left\{(c, u, v) \in \mathbf{C}^{3} \mid(u, v) \in V_{0}, c \in U\right\}$. One may suppose that $\partial f / \partial v$ is different from 0 in $V^{1}$. Then there exists the function $\nu_{v}$ for which $\partial f / \partial c+\partial f / \partial v \cdot \nu_{v} \equiv 0$ on $V^{1}$. Hence the vector field $\left(1,0, \nu_{v}\right)$ is tangent to the surface $H=\{(c, u, v) \mid f(c, u, v)=0\} \subset \mathbf{C}^{3}$. Let $\varphi_{t}$ be the phase flow associated with this field. Put $K_{0}=\bigcup_{i} \gamma_{i}^{0}$. Then $K_{t}=\varphi_{t}\left(K_{0}\right) \subset F_{t}$ for every arbitrarily small $t$. The set $K_{t}$ is the union of loops $\gamma_{i}^{t}=\varphi_{t}\left(\gamma_{i}^{0}\right)$, and we are under the assumptions of Lemma 2. For each fixed $t$ these loops belong to the same component of the curve $F_{t}$, since they have the common point $\phi_{t}\left(\omega_{0}\right)$. This concludes the proof of Lemma 3.

To each complex line $\left\{(u, v) \in \mathbf{C}^{2} \mid a u+b v+c=0\right\}$ in $\mathbf{C}^{2}$ one may assign the point $(a, b, c) \in \mathbf{C} \mathbf{P}^{2}$. Thus we can consider the set of complex lines in $\mathbf{C}^{2}$ as $W=\mathbf{C P}^{2}-(0,0,1)$ (the point $(0,0,1)$ has to be deleted since $a$ and $b$ cannot equal 0 simultaneously). Let the mapping $\rho: W \rightarrow \mathbf{C P}^{1}$ be given by the formula $(a, b, c) \rightarrow(a, b)$. Suppose that $P: M \rightarrow \mathbf{C}^{2}$ and $\Gamma$ are the same as above. Let $\Gamma^{\star}$ be the dual curve, i.e., $\Gamma^{\star}$ is the closure in $W$ of the set of points that correspond to tangent lines to $\Gamma$ at the smooth part of $\Gamma$. We shall denote the set of singular points of $\Gamma$ of $S$. For every $w \in \mathbf{C}^{2}$ we put $K(w)=\{\ell \in W \mid w \in \ell\}$ (here we consider $\ell$ as both a point in $W$ and as a line in $\mathbf{C}^{2}$ ) and $K=\bigcup_{w \in S} K(w) \subset W$. Let the curve $\Gamma$ be given by an equation $h(u, v)=0$ and let $\Lambda=\left\{\lambda_{i}=\left(a_{i}, b_{i}\right)\right\} \subset \mathbf{C P}^{1}$ be the set of roots of the leading homogeneous part of $h$. For each $\lambda \in \mathbf{C P}^{1}$ we put $L(\lambda)=\rho^{-1}(\lambda)$ and $L=\bigcup_{\lambda \in \Lambda} L(\lambda)$. Suppose that $P=(p, q)$ is the coordinate representation of $P$. Let $X$ be the closure (in the Euclidean topology of $W$ ) of the set of points $\{(a, b, c)\}$ such that the curve $\{z \in M \mid a p(z)+b q(z)+c=0\}$ is reducible.

Lemma 4. The set $X$ is contained in $\Gamma^{\star} \cup K \cup L$.

Proof. We may require that the polynomial $h$ does not have repeated factors. Put $m=\operatorname{deg} h$. Recall that a line $\ell$ is in general position relative to $\Gamma$, if the set $\ell \cap \Gamma$ consists of $m$ different points. By Lefschetz's theorem, for every line $\ell \subset \mathbf{C}^{2}$ in a general position the embedding $i: \ell \hookrightarrow \mathbf{C}^{2}$ induces an epimorphism $i_{\star}: \pi_{1}(\ell-\Gamma) \rightarrow \pi_{1}\left(\mathbf{C}^{2}-\Gamma\right)$ (see [Z] or [A]). Thus, by Lemma 1 , it is enough to check that if a line $\ell \notin \Gamma^{\star} \cup K \cup L$ then $\ell$ is in a general position. Let $\mathbf{C}^{2} \hookrightarrow \mathbf{C} \mathbf{P}^{2}$ be a natural embedding, $E=\mathbf{C P}^{2}-\mathbf{C}^{2}, \bar{\Gamma}$ be the 
closure of $\Gamma$ in $\mathbf{C P}^{2}$, and $\bar{\ell}$ be the closure of $\ell$ in $\mathbf{C P}^{2}$. Then the set $\bar{\ell} \cap \bar{\Gamma}$ consists of $m$ points for a line $\bar{l}$ in a general position. Note that if $\ell \notin L$ then $E \cap \bar{\ell} \cap \bar{\Gamma}=\varnothing$, i.e., $\bar{\ell} \cap \bar{\Gamma}=\ell \cap \Gamma \subset \mathbf{C}^{2}$. If $\ell \notin \Gamma^{*} \cup K$ as well, then $\ell$ meets $\Gamma$ transversally. Thus each $\ell \notin \Gamma^{\star} \cup K \cup L$ meets $\Gamma$ transversally at $m$ different points in $\mathbf{C}^{2}$.

Note that if $\rho(X)$ is a finite set, then for every $b$ such that $(1, b) \notin \rho(X)$ each fiber of the regular function $p(z)+b q(z)$ is irreducible. Since $\rho(L)=\Lambda$ is a finite set, the set $\rho(X)$ is finite in $X \cap\left(K \cup \Gamma^{\star}\right)$ is finite, or in other words, $\operatorname{dim} X \cap\left(K \cup \Gamma^{\star}\right)=0$.

Lemma 5. The set $X$ is algebraic.

Proof. Let $D$ be an irreducible component of $\Gamma^{\star} \cup K \cup L$. It suffices to prove that the set $D \cap X$ is algebraic. Consider the algebraic variety $B=\{(x, \ell) \mid x \in$ $\left.P^{-1}(\ell), \ell \in D\right\} \subset M \times W$. Let $\tau: B \rightarrow D$ be the natural projection, and let $n(c)$ be the number of irreducible components of a fiber $Q_{c}=\{x \mid(x, c) \in B\}$ of this mapping. We shall show later that the function $n(c)$ on $D$ coincides with a constant $n$ outside a finite set $C \subset D$. (Actually, a stronger fact holds. It is well-known that outside a finite subset of $D$ all fibers are diffeomorphic. For our purpose it suffices to check the simpler assertion.) If $n>1$ then $X \supset D-C$, i.e., $X \cap D=D$. Otherwise $X \cap D \subset C$ and $X \cap D$ is again an algebraic variety. Now we have to show that the set $C$ is finite. Let $B^{\prime}$ be the smooth part of $B$. The algebraic variety $A=B-B^{\prime}$ is the union of a finite number of irreducible algebraic curves and points. Now we define the set $D_{0} \subset D$ such that for $c \in D_{0}$ an irreducible component of the set $A$ belongs to the fiber $Q_{c}$. Hence the set $D_{0}$ is finite. Note that the number of components of $Q_{c}^{\prime}=Q_{c} \cap B^{\prime}$ coincides with the number of components of $Q_{c}$, when $c \notin D_{0}$. Consider a smooth compact algebraic curve $\bar{D} \supset D-D_{0}$. Put $\phi=\left.\tau\right|_{B^{\prime}}$. Standard results from the theory of resolution of singularities yield the existence of a smooth compact algebraic variety $\bar{B} \supset B^{\prime}$ for which there exists an extension $\bar{\phi}: \bar{B} \rightarrow \bar{D}$ of the mapping $\phi$. Let $B_{1}$ be the subset of points in $\bar{B}$ for which the mapping $\bar{\phi}$ is not smooth. Then $D_{1}=\bar{\phi}\left(B_{1}\right)$ is a finite algebraic subvariety of $\bar{D}$ [M, Proposition 3.7]. The proper mapping $\bar{\phi}: \bar{B}-\bar{\phi}^{-1}\left(D_{1}\right) \rightarrow \bar{D}-D_{1}$ is smooth at every point and, therefore, it is a smooth fibration [MK, Chapter 1, Theorem 4.1]. In particular, the number of irreducible components of the curve $\bar{Q}_{c}=\bar{\phi}^{-1}(c)$ is constant when $c \notin D_{1}$. The set $A^{\prime}=\bar{B}-B^{\prime}$ is an algebraic curve. Let $D_{2}$ be the subset of points $c \in \bar{D}$ such that $\bar{\phi}(E)=c$ for a certain irreducible component $E$ of the curve $A^{\prime}$. Since the number of irreducible components of the curve $A^{\prime}$ is finite, the set $D_{2}$ is finite. Put $C^{\prime}=D_{1} \cup D_{2}$. If $c \notin C^{\prime}$ then the set $\bar{Q}_{c}-Q_{c}^{\prime}$ is finite. Thus the curves $\bar{Q}_{c}$ and $Q_{c}^{\prime}$ have the same number of irreducible components. The algebraic set $\bar{D}-\left(D-D_{0}\right)$ is finite.

Lemma 6. Let $\ell$ be a $\Gamma$ proper line in $K(w)$ for some point $w$. The dimension of $X \cap K(w)=0$.

Proof. One may suppose that $w$ coincides with the origin and that $\ell$ is $\{u=$ $0\}$. Let $\ell_{c}=\{(u, v) \mid u+c v=0\}$. By Lemma 3, we have the epimorphism $i_{\star}^{c}: \pi_{1}\left(\ell_{c}-\Gamma\right) \rightarrow \pi_{1}\left(\mathbf{C}^{2}-\Gamma\right)$, when $|c|$ is sufficiently small. By Lemma 1 this 
implies that $P^{-1}\left(\ell_{c}\right)$ is irreducible. Since $X \cap K(w)$ is an algebraic variety, there can be only finite number of complex numbers $\left\{c_{k}\right\}$ such that the line $\ell_{c_{k}} \in K$. Thus $\operatorname{dim} X \cap K(w)=0$.

Note that if $\Gamma$ is reducible then $\Gamma^{\star}$ is the union of the duals of its reducible components.

Lemma 7. Let $\Gamma_{i}$ be an irreducible component of $\Gamma$, let $\Gamma_{i}^{\star}$ be the dual curve for $\Gamma_{i}$, and let $l$ be a tangent line to $\Gamma_{i}$ at a regular point $w_{i}$. If $\ell$ is $\Gamma$ proper then $\operatorname{dim} X \cap \Gamma_{i}^{\star}=0$.

Proof. We may again suppose that $w$ is the origin and $\ell$ is $\{u=0\}$. The curve $\Gamma_{i}$ is locally given by the equation $u=u(v)=a v^{n}+v^{n+1} h(v)$, where $a \neq 0$ and $n \geq 2$. For every point $(u(c), c) \in \Gamma_{i}$ when $|c|$ is sufficiently small, we have the following equation for the tangent line to $\Gamma_{i}$ at the point

$$
u-n a c^{n-1} v+(n-1) a c^{n}+v \cdot O\left(|c|^{n}\right)+O\left(|c|^{n+1}\right)=0 .
$$

Since $X \cap \Gamma_{i}^{\star}$ is an algebraic variety, application of Lemma 3 again provides the desired conclusion.

Let $A_{g}: \mathrm{C}^{2} \rightarrow \mathrm{C}^{2}$ be a polynomial automorphism given by the formula

$$
A_{g}(u, v)=(u+\dot{g}(v), v),
$$

where $g \in \mathbf{C}[v]$ is a polynomial in one variable. Put $P_{g}=A_{g} \circ P$, and let the symbols $\Gamma_{g}, \Gamma_{i g}, \Gamma_{g}^{\star}, \Gamma_{i g}^{\star}, X_{g}, K_{g}(w), K_{g}, L_{g}, S_{g}$ have the same meaning for the mapping $P_{g}$ as the symbols $\Gamma, \Gamma_{i}, \Gamma^{\star}, \Gamma_{i}^{\star}, X, K(w), K, L, S$ for $P$.

Lemma 8. Let the line $\{v=1\}$ be $\Gamma$ proper. Then there exists a polynomial $g \in \mathbf{C}[v]$ satisfying

(1) for every irreducible component $\Gamma_{i g} \subset \Gamma_{g}$ there is a regular point $w_{i}^{\prime} \in$ $\Gamma_{i g}$ such that the tangent line $\ell_{i}^{1}$ to $\Gamma_{i g}$ at $w_{i}^{\prime}$ is $\Gamma_{g}$ proper,

(2) for every $z_{j}^{\prime} \in S_{g}$ there exists a line $l_{j}^{2} \in K_{g}\left(z_{j}^{\prime}\right)$ that is $\Gamma_{g}$ proper.

Proof. Choose $w_{i} \in \Gamma_{i}$ so that the tangent line to $\Gamma_{i}$ at $w_{i}$ is given by the equation $u+b_{i}^{1} v+d_{i}^{1}=0$, where $b_{i}^{1}, d_{i}^{1} \in \mathbf{C}$. Let $V$ be the set of the $v$ coordinates of the points of the set $S \cup\left\{w_{i}\right\}$. Choose a polynomial $h \in \mathbf{C}[v]$ so that for every $v_{0} \in V$ we have

$$
h\left(v_{0}\right)=\frac{d h}{d v}\left(v_{0}\right)=0 .
$$

Put $g(v)=c(v-1) \cdot h(v)$, where $c$ is an arbitrarily large number. Suppose that a line $\ell_{j}^{2}=\left\{u+b_{j}^{2} v+d_{j}^{2}=0\right\}$ belongs to $K\left(z_{j}\right)$, where $z_{j} \in S$. Choose a line $\ell$ given by the equation $\{a u+b v+d=0\}$. Then the curve $\ell_{g}=A_{g}^{-1}(\ell)$ coincides with

$$
a u+a g(v)+b v+d=0 .
$$

Vice versa: for each curve $\ell_{g}$ given by the equation (2) the curve $A_{g}\left(\ell_{g}\right)$ is a complex line. For $k=1,2$ put $\ell_{g}^{k i}=\left\{(u, v) \mid u+g(v)+b_{i}^{k} v+d_{i}^{k}=\right.$ $\left.u+b_{i}^{k} v+d_{i}^{k}+c(v-1) h(v)=0\right\}$. By (1), the curve $\ell_{g}^{1 i}$ is tangent to $\Gamma_{i}$ at $w_{i}$. 
Hence $A_{g}\left(\ell_{g}^{1 i}\right)$ is tangent to $A_{g}\left(\Gamma_{i}\right)$ at $w_{i}^{\prime}=A_{g}\left(w_{i}\right)$. Note that $A_{g}\left(\Gamma_{i}\right)=\Gamma_{i g}$, $A_{g}(\Gamma)=\Gamma_{g}$, and the equation of the curve $\ell_{g}^{k i}$ can be written in the form

$$
c^{-1}\left(u+b_{i}^{k} v+d_{i}^{k}\right)+(v-1) h(v)=0 .
$$

Recall that the line $\{v=1\}$ is $\Gamma$ proper. Application of Lemma 3 shows that $\ell_{g}^{k i}$ is $\Gamma$ proper. Hence each line $l_{i}^{k}=A_{g}\left(\ell_{g}^{k i}\right)$ is $\Gamma_{g}$ proper. It remains to note that $S_{g}=A_{g}(S)$. This concludes the proof of Lemma 8 .

Let $P=(p, q): \mathbf{C}^{2} \rightarrow \mathbf{C}^{2}$ have the Jacobian $J(p, q) \equiv 1$. Using an affine automorphism of $\mathbf{C}^{2}$ if necessary, one may suppose that the line $\{v=1\}$ is $\Gamma$ proper. Let $A_{g}$ be the same as in Lemma 8. Then $P$ is invertible iff $P_{g}=A_{g} \circ P$ is invertible. Keeping the previous notation, one may assert (by Lemma 7) that $\operatorname{dim} X_{g} \cap\left(K_{g} \cup \Gamma_{g}^{\star}\right)=0$. Thus $\rho\left(X_{g}\right)$ is a finite set. If a point $(1, b) \notin \rho\left(X_{g}\right)$ and $P_{g}=\left(p_{1}, q_{1}\right)$ is the coordinate representation of $P_{g}$, then the polynomial $p_{1}+b q_{1}$ has irreducible fibers only. The theorem is proved.

\section{ACKNOWLEDGMENTS}

It is a pleasure to thank V. Lin, S. Orevkov, and M. Zaidenberg for useful discussions and advice. The author is grateful to the referee for valuable remarks.

\section{REFERENCES}

[A] S. Abhyankar, Über die endliche Erzeugung der Fundamentalgruppe einer kimplex-algebraischen Mannigfaltigkeit, Math. Ann. 139 (1960), 265-274.

[BCW] H. Bass, E. H. Connell and D. Wright, The Jacobian Conjecture: Reduction of degree and formal expansion of inverse, Bull. Amer. Math. Soc. 7 (1982), 287-300.

[F] E. Formanek, Two notes on the Jacobian Conjecture, Arch. Math. (Basel) 49 (1987), 286291.

[FR] D. B. Fuks and V. A. Rokhlin, Beginner's course in topology. Geometric chapters, SpringerVerlag, Berlin, Heidelberg, New York, and Tokyo, 1984.

[K] O.-H. Keller, Ganze Gremona-transformation, Monatsch. Math. Phys. 47 (1939), 299-306.

[M] D. Mumford, Algebraic geometry, Complex projective varieties, Springer-Verlag, Berlin, Heidelberg and New York, 1976.

[MK] J. Morrow and K. Kodaira, Complex manifolds, Holt, Rinehart, and Winston Inc., New York, Chicago, San Francisco, Atlanta, Dallas, Montreal, Toronto, London, and Sydney, 1971.

[NR] W. D. Neumann and L. Rudolph, Unfoldings in knot theory, Math. Ann. 278 (1987), 409439; Corrigendum, Unfoldings in knot theory, Math. Ann. 282 (1988), 349-351.

[R] M. Razar, Polynomial maps with constant Jacobian, Israel Math. J. 32 (1979), 97-106.

[S1] Y. Stein, On density of image of differential operators generated by polynomials, J. Analyse Math. 52 (1989), 291-300.

[S2] Linear differential operators related to the Jacobian Conjecture have a closed image, J. Analyse Math. 54 (1990), 237-245.

[S3] _ On linear differential operators related to the Jacobian Conjecture, J. Pure Appl. Algebra 57 (1989), 175-186. 
[W] S. S. Wang, A generalization of a lemma of Abhyankar and Moh, J. Pure Appl. Algebra 40 (1986), 297-299.

[Z] O. Zariski, On the problem of existence of algebraic functions of two variables possessing a given branch curve, Amer. J. Math. 51 (1929), 305-328.

Department of Mathematics, Wayne State University, Detroit, Michigan 48202

Current address: Department of Mathematics and Computer Science, University of Miami, Coral Gables, Florida 33124

E-mail address: kaliman@paris.cs.miami.edu 